\title{
TIME REVERSAL: ALGORITHMS FOR M-ARY TARGET CLASSIFICATION USING ARRAY SIGNAL PROCESSING
}

\author{
Foroohar Foroozan and Amir Asif \\ Computer Science and Engineering \\ York University, Toronto, ON, Canada
}

\begin{abstract}
An $M$-ary time reversal (TR) maximum likelihood classifier for a single pair of transmitting and receiving transducer element was derived in [1] for underwater acoustic target detection applications. This paper considers a more general TR setup consisting of a $P$-element transmitting array and an $N$ element receiving array and derives the $M$-ary conventional and TR classifiers for the multielement case in an electromagnetic communication environment. We show that the TR algorithm provides a classification gain of over $3 \mathrm{~dB}$ at low signal to noise ratios as compared to the conventional classifiers.
\end{abstract}

\section{INTRODUCTION}

At low signal to noise ratios, backscattered signals from targets are weak and distorted by clutter and multipaths, which confounds detection, causes erratic tracking, and makes it difficult to extract useful information from noisy observations for imaging and classification applications in digital communications. TR provides a simple methodology to adapt the transmitted waveform to the channel and offers a unique paradigm that constructively utilizes multipaths to its advantage while mitigating effects of other distortions [2]- [4].

In TR, the channel is illuminated with a probing signal. The backscatters of the probing signal is recorded by a receiving array of antennas, energy normalized, and retransmitted back (physically or synthetically) into the medium in the reverse temporal chronology. The final backscatters of the time reversed signals are used by the TR classifiers to enhance the performance of the TR detectors. The impact of multipath/scattering richness on the TR detection performance can be attributed intuitively to the tighter refocusing, referred to as super-resolution focusing that beats the

This work was supported in part by the Natural Science and Engineering Research Council (NSERC), Canada under Grant No. 2284152007.

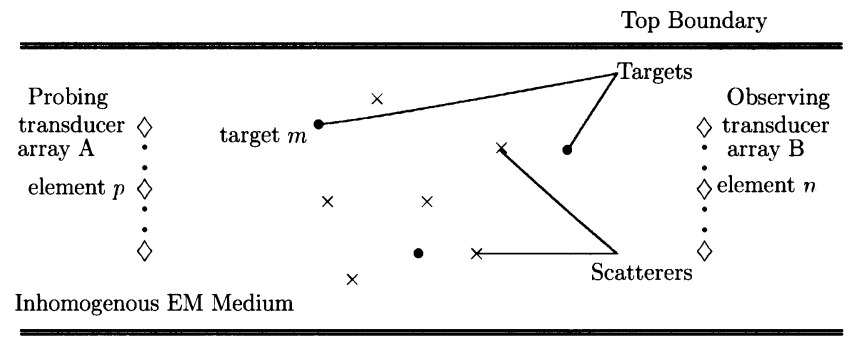

Bottom Boundary

Fig. 1. Experimental setup for the TR classification algorithms. Array $A$, shown on the left hand side, consists of $P$ transducer elements and is used to probe the channel. Array $B$, shown on the right, consists of $N$ transducer elements and receives the backscatter of the probing signal. The backscatter is energy normalized, time reversed, and retransmitted back into the channel by Array $B$. The final backscatter of the time reversed signal, as recorded by Array $A$, is used by the TR classifier. Symbol $\bullet$ represents the targets (objects of interest), while symbol $\times$ represents the unwanted scatterers.

Rayleigh diffraction limit. Intuitively, multipaths increase the effective array aperture resulting in tighter focusing that leads to an improvement in the detection performance. Currently, there is a growing research effort to apply time reversal techniques using electromagnetic (EM) waves. As the RF instruments become more available, the TR methods find applications in wireless communications, remote sensing systems, microwave medical imaging, as well as in radar detection and imaging. This paper designs TR detectors for EM radar and communications applications, considering the $M$-ary hypothesis problem [5]

$$
\begin{array}{cl}
\mathbb{H}_{0}: & \text { No target is present, } \\
\mathbb{H}_{i}: & \text { Target } i,(1 \leq i \leq(M-1)), \text { is present, }
\end{array}
$$

using multielement antenna arrays based on the experimental setup shown in Fig. 1. In other words, we are interested in detecting the presence of the target and classifying the detected target in one of the known $(M-1)$ categories. The algorithms derived in this paper are generalizations of the earlier results presented in [1] and [6]. Reference [1] con- 
straints both the transmitting and receiving antenna arrays individually to a pair of single transducer elements, while reference [6] considers a binary detection problem. The paper uses an experimental setup consisting of a $P$-element transmitting array and an $N$-element receiving array to solve the $M$-ary classification problem. In contrast to the earlier work, where we used the wave hydrodynamic equation to model the propagation wave in an acoustic environment, this paper considers an EM domain simulated using the Finite Difference Time Domain (FDTD) approach [7].

The performance of the $M$-ary classifier is compared with that of the conventional classification algorithms, which are based only on the observed backscatter of the probing signal used in the forward propagation step. The TR classifier, on the other hand, uses the backscatter of the time reversed signal. The final observations of both the conventional and TR classifiers are normalized to have unit energy before being used by the respective classifiers. The TR classifier uses the reciprocity theorem [8] such that the position of a point source and observation site can be reversed without altering the observation itself even in a lossless, inhomogeneous medium. Experimental results verify the superior performance of the TR classifiers in the EM domain. At low signal to noise ratios (SNR), the TR classifier outperforms the conventional classifier by over $3 \mathrm{~dB}$ in our simulations.

The rest of the paper is organized as follows. After introducing the notation in Section 2, Section 3 derives both the $M$-ary conventional and TR classifiers for multielement transducer arrays. In Section 4, the special case of single element transmitter-receiver arrays is considered. The performance of the $M$-ary TR classifier is compared with the conventional classifier in Section 5. Finally, Section 6 concludes the paper.

\section{PROBLEM FORMULATION}

In this section, we introduce our notation based on Fig. 1, which illustrates the general setup for time reversal using a pair of antenna arrays. Array $A$ consists of $(1 \leq p \leq P)$ transducer elements and is used initially to probe the channel. The probing signal $F_{p}\left(\omega_{q}\right)$, transmitted by element $p$ of Array $A$, is reflected collectively by the targets (shown in Fig. 1 as ' $\bullet$ ') and scatterers (shown as ' $X$ ') such that individual observations are recorded by $(1 \leq n \leq N)$ elements of Array $B$. Our classification algorithm uses background subtraction [6]

$$
\mathbf{H}_{t_{i}}\left(\omega_{q}\right)=\mathbf{H}_{c+t_{i}}\left(\omega_{q}\right)-\mathbf{H}_{c}\left(\omega_{q}\right)
$$

to eliminate the effect of clutter in the channel response matrix. In Eq. (1), notation $\mathbf{H}_{c}\left(\omega_{q}\right)$ refers to the $(N \times P)$ clutter response matrix defined as

$$
\begin{array}{r}
\forall p=1, \cdots, P \quad \text { and } \quad \forall n=1, \cdots, N \\
\mathbf{H}_{c}\left(\omega_{q}\right) \triangleq\left\{H_{c}\left(\omega_{q} ; B_{n} \leftarrow A_{p}\right)\right\},
\end{array}
$$

where $H_{c}\left(\omega_{q} ; B_{n} \leftarrow A_{p}\right)$ is the channel response observed at element $n$ of Array $B$ when element $p$ of Array $A$ probes the channel with a delta function during the training phase when none of the targets are present. Similarly, notation $\mathbf{H}_{c+t_{i}}\left(\omega_{q}\right)$ in Eq. (1) denotes the clutter/target multistatic matrix in the presence of target $i$ and the unwanted scatterers. The independent variable $\omega_{q}$ denotes discretized frequency. The target data matrix defined in Eq. (1) contains both the direct reflection between the target and the receive array, and the secondary reflections between the scatterers, the target, and the receive array. The background subtraction eliminates the effect of clutters in the target channel response, the interactions between the target and scatterers are ignored in this work.

In terms of the multistatic matrices, the backscatter observations of the probing signal $F_{p}\left(\omega_{q}\right)$ are given by

$$
\mathbf{r}_{(i, p)}^{(l)}\left(\omega_{q}\right)=\mathbf{H}_{t_{i}}\left(\omega_{q}\right) \mathbf{e}_{p} F_{p}\left(\omega_{q}\right)+\mathbf{v}_{(i, p)}^{(l)}\left(\omega_{q}\right),
$$

for $(1 \leq p \leq P)$. Notation $\mathbf{e}_{p}$ is a $(P \times 1)$ vector of zero entries except the $p$ 'th column, which is 1 . The subscript $(i, p)$ in the observation $\mathbf{r}_{(i, p)}^{(l)}\left(\omega_{q}\right)$ signifies that element $p$ of the Array $A$ probes the channel and the backscatter results due to target $i$. Variable $\mathbf{v}_{(i, p)}^{(l)}\left(\omega_{q}\right)$ represents the observation noise vector comprising of independent identically distributed (iid) random entries. The probing step is repeated $(1 \leq l \leq L)$ times and the superscript used in Eq. (3) represents the l'th observation. The cumulative received signals at Array $B$ for the l'th snapshot, when all elements of Array $A$ probe the channel together with $F_{p}\left(\omega_{q}\right)$, is, therefore, given by

$$
\mathbf{r}_{(i)}^{(l)}\left(\omega_{q}\right) \triangleq \sum_{p=1}^{P} \mathbf{r}_{(i, p)}^{(l)}\left(\omega_{q}\right)=\mathbf{H}_{t_{i}}\left(\omega_{q}\right) \mathbf{f}\left(\omega_{q}\right)+\mathbf{v}_{(i)}^{(l)}\left(\omega_{q}\right)
$$

where $\mathbf{v}_{(i)}^{(l)} \sim \mathcal{C N}\left(\mathbf{0}, \sigma_{v}^{2} \mathbf{I}_{N}\right)$ is accumulated noise, and

$$
\mathbf{f}\left(\omega_{q}\right)=\left[F_{1}\left(\omega_{q}\right), \cdots, F_{P}\left(\omega_{q}\right)\right]^{T}
$$

is the probing signal vector. Conventional classifiers use observations $\mathbf{r}_{(i)}^{(l)}\left(\omega_{q}\right)$ obtained from the forward probing step for detection/classification of the targets.

Following the principle of TR, the recorded signal $\mathbf{r}_{(i)}^{(l)}\left(\omega_{q}\right)$ is energy normalized, time reversed (equivalent 
to phase conjugation in the frequency domain), and retransmitted back into the medium. After subtraction of the clutter response, the backscattered TR signal at Array $A$ is given by

$$
\begin{aligned}
\mathbf{p}_{i}^{(l)}\left(\omega_{q}\right) & =c_{i}^{(l)} \mathbf{H}_{t_{i}}^{T}\left(\omega_{q}\right) \mathbf{r}_{(i)}^{(l) *}\left(\omega_{q}\right)+\mathbf{w}_{i}^{(l)}\left(\omega_{q}\right) \\
& =c_{i}^{(l)} \mathbf{H}_{t_{i}}^{T}\left(\omega_{q}\right)\left[\mathbf{H}_{t_{i}}^{*}\left(\omega_{q}\right) \mathbf{f}^{*}\left(\omega_{q}\right)+\mathbf{v}_{(i)}^{(l) *}\left(\omega_{q}\right)\right] \\
& +\mathbf{w}_{i}^{(l)}\left(\omega_{q}\right)
\end{aligned}
$$

where $\mathbf{w}_{i}^{(l)}\left(\omega_{q}\right) \sim \mathcal{C N}\left(\mathbf{0}, \sigma_{w}^{2} \mathbf{I}_{p}\right)$ denotes the observation noise during the TR step. In Eq. (6), we exploit the fact that the target channel response from Array $B$ to Array $A$ is $\mathbf{H}_{t_{i}}^{T}\left(\omega_{q}\right)$ due to the reciprocity property of the channel. Notation $c_{i}^{(l)}$ represents the signal gain used in the energy normalization step prior to time reversal. Using Eqs. (4) and (6), the statistics of the forward backscattered signal $\mathbf{r}_{(i)}^{(l)}\left(\omega_{q}\right)$ and TR backscattered signal $\mathbf{p}_{i}^{(l)}\left(\omega_{q}\right)$ can be derived as

$$
\begin{aligned}
\mathbf{r}_{(i)}^{(l)}\left(\omega_{q}\right) & \sim \mathcal{C N}\left(\mathbf{H}_{t_{i}}\left(\omega_{q}\right) \mathbf{f}\left(\omega_{q}\right), \sigma_{v}^{2} \mathbf{I}_{N}\right) \\
\mathbf{p}_{(i)}^{(l)}\left(\omega_{q}\right) & \sim \mathcal{C N}\left(c_{i}^{(l)} \mathbf{H}_{t_{i}}^{T}\left(\omega_{q}\right) \mathbf{H}_{t_{i}}^{*}\left(\omega_{q}\right) \mathbf{f}^{*}\left(\omega_{q}\right), \Sigma\left(\omega_{q}\right)\right),
\end{aligned}
$$

where the covariance matrix $\Sigma\left(\omega_{q}\right)$ is given by

$$
\Sigma\left(\omega_{q}\right)=c_{i}^{(l)^{2}} \mathbf{H}_{t_{i}}^{T}\left(\omega_{q}\right) \mathbf{H}_{t_{i}}^{*}\left(\omega_{q}\right) \sigma_{v}^{2}+\sigma_{w}^{2} \mathbf{I}_{p} .
$$

Next, we derive the maximum likelihood functions for the conventional and TR classifiers.

\section{M-ARY ML CLASSIFIERS USING MULTIELEMENT ARRAYS}

In this section, we derive: $(i)$ the conventional $M$-ary maximum likelihood (ML) classifier, which uses only the forward backscatters $\mathbf{r}_{(i)}^{(l)}\left(\omega_{q}\right)$; and (ii) the TR classifier, which uses the TR observations $\mathbf{p}_{(i)}^{(l)}\left(\omega_{q}\right)$ in addition to the forward backscatters. Our classification algorithms specify if a target is present and to which of the $(M-1)$ classes does the detected target belongs. It assumes target signatures $\mathbf{H}_{t_{i}}\left(\omega_{q}\right)$ are all known. Based on Eq. (7), the conditional probability density function (pdf) of the zero mean observations

$$
\overline{\mathbf{r}}_{(i)}^{(l)}\left(\omega_{q}\right)=\mathbf{r}_{(i)}^{(l)}\left(\omega_{q}\right)-\mathbf{H}_{t_{i}}\left(\omega_{q}\right) \mathbf{f}\left(\omega_{q}\right),
$$

observed during the forward probing step, is given by

$$
\begin{aligned}
& \operatorname{Pr}\left(\overline{\mathbf{r}}_{(i)} \mid \mathbb{H}_{i}\right)= \\
& \prod_{l=1}^{L} \prod_{q=0}^{Q-1} \frac{1}{\pi\left|\Omega\left(\omega_{q}\right)\right|} \exp \left\{-\overline{\mathbf{r}}_{(i)}^{(l)}\left(\omega_{q}^{H}\right) \Omega^{-1}\left(\omega_{q}\right) \overline{\mathbf{r}}_{(i)}^{(l)}\left(\omega_{q}\right)\right\}
\end{aligned}
$$

Using the following log identity

$$
\ell_{i}\left(\mathbf{r}_{(i)}\right)=\ln \left(\operatorname{Pr}\left(\mathbf{r}_{(i)} \mid \mathbb{H}_{i}\right)\right)-\ln \left(\operatorname{Pr}\left(\mathbf{r}_{(i)} \mid \mathbb{H}_{0}\right)\right),
$$


the log likelihood ratio is given by

$$
\begin{aligned}
\ell_{i}\left(\mathbf{r}_{(i)}\right) & =\frac{1}{\sigma_{v}^{2}} \sum_{l=1}^{L} \sum_{q=0}^{Q-1}\left[2 \Re\left\{\left(\mathbf{H}_{t_{i}}\left(\omega_{q}\right) \mathbf{f}\left(\omega_{q}\right)\right)^{H} \mathbf{r}_{(i)}^{(l)}\left(\omega_{q}\right)\right\}\right. \\
& \left.-\left\|\mathbf{H}_{t_{i}}\left(\omega_{q}\right) \mathbf{f}\left(\omega_{q}\right)\right\|^{2}\right]
\end{aligned}
$$

Ignoring the second term which is a constant, and normalizing the result with 2|| $\mathbf{H}_{t_{i}}\left(\omega_{q}\right) \mathbf{f}\left(\omega_{q}\right) \| / \sigma_{v}$, the likelihood ratio $\ell_{i}\left(\mathbf{r}_{(i)}\right)$ in Eq. (18) simplifies to Theorem 1.

In order to derive the mean, we take the expected value of $\ell_{i}\left(\mathbf{r}_{(i)}\right)$ specified in Theorem 1. Substituting the expected value of $\mathbf{r}_{(i)}$ from Eq. (7), we get

$$
\mu_{i}=\sum_{q=0}^{Q-1} \Re\left(\frac{L\left(\mathbf{H}_{t_{i}}\left(\omega_{q}\right) \mathbf{f}\left(\omega_{q}\right)\right)^{H}\left(\mathbf{H}_{t_{i}}\left(\omega_{q}\right) \mathbf{f}\left(\omega_{q}\right)\right)}{\sigma_{v}\left\|\mathbf{H}_{t_{i}}\left(\omega_{q}\right) \mathbf{f}\left(\omega_{q}\right)\right\|}\right)
$$

which simplifies to $\sum_{q=0}^{Q-1} L\left\|\mathbf{H}_{t_{i}}\left(\omega_{q}\right) \mathbf{f}\left(\omega_{q}\right)\right\| / \sigma_{v}$.

For the variance, we note that the only random term in $\ell_{i}\left(\mathbf{r}_{(i)}\right)$ specified in Theorem 1, is $\sum_{l=1}^{L} \mathbf{r}_{(i)}^{(l)}\left(\omega_{q}\right)$. Assuming independent observations and substituting the value of the variance of $\mathbf{r}_{(i)}$ from Eq. (7), we have

$$
\begin{aligned}
& \operatorname{var}\left\{\ell_{i}\left(\mathbf{r}_{(i)}\right)\right\}= \\
& \qquad \Re\left(\frac{L \sigma_{v}^{2} \sum_{q=0}^{Q-1}\left(\mathbf{H}_{t_{i}}\left(\omega_{q}\right) \mathbf{f}\left(\omega_{q}\right)\right)^{H}\left(\mathbf{H}_{t_{i}}\left(\omega_{q}\right) \mathbf{f}\left(\omega_{q}\right)\right)}{\sigma_{v}^{2} \sum_{q=0}^{Q-1}\left\|\mathbf{H}_{t_{i}}\left(\omega_{q}\right) \mathbf{f}\left(\omega_{q}\right)\right\|^{2}}\right),
\end{aligned}
$$

which simplifies to $L / 2$.

Theorem 2. The M-ary log likelihood ratio of $\mathbf{p}_{(i)}^{(l)}\left(\omega_{q}\right)$ of the TR classifier using multielement antenna arrays $A$ and $B$ is given by

$$
\begin{aligned}
& \ell_{i}\left(\mathbf{p}_{(i)}\right)= \\
& \sum_{q=0}^{Q-1} \Re\left(\frac{\left(\mathbf{H}_{t_{i}}^{T}\left(\omega_{q}\right) \mathbf{H}_{t_{i}}^{*}\left(\omega_{q}\right) \mathbf{f}^{*}\left(\omega_{q}\right)\right)^{H} \sum_{l=1}^{L} \mathbf{p}_{(i)}^{(l)}\left(\omega_{q}\right)}{\sigma_{w}\left\|\mathbf{H}_{t_{i}}^{T}\left(\omega_{q}\right) \mathbf{H}_{t_{i}}^{*}\left(\omega_{q}\right) \mathbf{f}^{*}\left(\omega_{q}\right)\right\|}\right),
\end{aligned}
$$

with the following conditional pdf's

$$
\begin{aligned}
\ell_{i}\left(\mathbf{r}_{(i)}\right) \mid \mathbb{H}_{0} & \sim \mathcal{C N}(0, L / 2) \\
\ell_{i}\left(\mathbf{r}_{(i)}\right) \mid \mathbb{H}_{i} & \sim \mathcal{C N}\left(\nu_{i}, L / 2\right)
\end{aligned}
$$

where $\nu_{i}=\sum_{q=0}^{Q-1} \frac{L c\left\|\mathbf{H}_{t_{i}}^{T}\left(\omega_{q}\right) \mathbf{H}_{t_{i}}^{*}\left(\omega_{q}\right) \mathbf{f}_{A}^{*}\left(\omega_{q}\right)\right\|}{\sigma_{w}}$.

Proof. Substituting $\boldsymbol{\Gamma}\left(\omega_{q}\right)=\sigma_{w} \mathbf{I}_{P}$ and $\overline{\mathbf{p}}_{(i)}^{(l)}\left(\omega_{q}\right)$ form
Eq. (11) in Eq. (12), we get

$$
\begin{aligned}
& \operatorname{Pr}\left(\mathbf{p}_{(i)} \mid \mathbb{H}_{i}\right)= \\
& \prod_{l=1}^{L} \prod_{q=0}^{Q-1} \frac{1}{\pi\left|\Gamma\left(\omega_{q}\right)\right|} \exp \left\{-\frac{\left\|\mathbf{p}_{(i)}\left(\omega_{q}\right)\right\|^{2}}{\sigma_{w}^{2}}\right. \\
& +\frac{2 \Re\left\{\left(\mathbf{H}_{t_{i}}^{T}\left(\omega_{q}\right) \mathbf{H}_{t_{i}}^{*}\left(\omega_{q}\right) \mathbf{f}^{*}\left(\omega_{q}\right)\right)^{H} \mathbf{p}_{(i)}\left(\omega_{q}\right)\right\}}{\sigma_{w}^{2}} \\
& \left.-\frac{\left.\| c_{i}^{(l)} \mathbf{H}_{t_{i}}^{T}\left(\omega_{q}\right) \mathbf{H}_{t_{i}}^{*}\left(\omega_{q}\right) \mathbf{f}^{*}\left(\omega_{q}\right)\right) \|^{2}}{\sigma_{w}^{2}}\right\} .
\end{aligned}
$$

When no target is present, we set $\mathbf{H}_{t_{i}}$ to zero in Eq. (19), therefore, $\operatorname{Pr}\left(\mathbf{p}_{(i)} \mid \mathbb{H}_{0}\right)$ is given by

$$
\begin{aligned}
& \operatorname{Pr}\left(\mathbf{p}_{(i)} \mid \mathbb{H}_{0}\right)= \\
& \quad \prod_{l=1}^{L} \prod_{q=0}^{Q-1} \frac{1}{\pi\left|\Gamma\left(\omega_{q}\right)\right|} \exp \left\{-\frac{\left\|\mathbf{p}_{(i)}\left(\omega_{q}\right)\right\|^{2}}{\sigma_{w}^{2}}\right\} .
\end{aligned}
$$

Here we assume that all the normalization constants $c_{i}^{(l)}$ are equal to $c$. Using the log identity given in Eq. (17), ignoring the constant terms, and normalizing the result with $2 c|| \mathbf{H}_{t_{i}}^{T}\left(\omega_{q}\right) \mathbf{H}_{t_{i}}^{*}\left(\omega_{q}\right) \mathbf{f}^{*}\left(\omega_{q}\right) \| / \sigma_{w}$, the result simplifies to Theorem 2.

The expressions for the mean and variance are derived by following the same steps used in deriving the mean and variance of the conventional classifier in the proof of Theorem 1 .

\section{SPECIAL CASE: \\ SINGLE TRANSMITTER-RECEIVER PAIR}

In [1], a special case of the ML classifiers for a single $(P=$ 1) element transmitter array and a single $(N=1)$ element receiver array was presented. In this section, we show that Theorems 1 and 2 reduce to the results presented in [1]. Corollary 1 corresponds to the special case for the conventional classifier (Theorem 1), while Corollary 2 corresponds to the TR classifier (Theorem 2). Note that the notations used in Corollaries 1 and 2 are different as explained in their proofs.

Corollary 1. The test statistics $\ell_{i}\left(\mathbf{r}_{i}\right)$ of the $M$-ary conventional classifier based on the observation vector $\mathbf{r}_{i}$ for a single transmitter-receiver pair is given by

$$
\ell_{i}\left(\mathbf{r}_{i}\right)=\Re\left(\frac{\left(\mathbf{F h}_{t_{i}}\right)^{H} \sum_{l=1}^{L} \mathbf{r}_{i}^{(l)}}{\sigma_{v}\left\|\mathbf{F} \mathbf{h}_{t_{i}}\right\|}\right)
$$

which is linear with the following conditional pdf's.

$$
\begin{aligned}
& \ell_{i}\left(\mathbf{r}_{i}\right) \mid \mathbb{H}_{0} \sim \mathcal{C N}(0, L / 2) \\
& \forall(i \neq 0), \quad \ell_{i}\left(\mathbf{r}_{i}\right) \mid \mathbb{H}_{i} \sim \mathcal{C N}\left(\mu_{i}, L / 2\right)
\end{aligned}
$$


where mean $\mu_{i}$ equals $L\left\|\mathbf{F h}_{t_{i}}\right\| / \sigma_{v}$.

Proof. Since $P=N=1$, the target channel frequency response $H_{t_{i}}\left(\omega_{q}\right)$ is just a scalar, therefore, Eq. (3) can be represented in the scalar form as

$$
r_{(i)}^{(l)}\left(\omega_{q}\right)=H_{t_{i}}\left(\omega_{q}\right) F\left(\omega_{q}\right)+v_{(i)}^{(l)}\left(\omega_{q}\right) .
$$

The expression for the log likelihood ratio given in Theorem 1 , simplifies to

$\ell_{i}\left(r_{(i)}\right)=\sum_{q=0}^{Q-1} \Re\left(\frac{\left(H_{t_{i}}\left(\omega_{q}\right) F\left(\omega_{q}\right)\right)^{H} \sum_{l=1}^{L} r_{(i)}^{(l)}\left(\omega_{q}\right)}{\sigma_{v}\left\|H_{t_{i}}\left(\omega_{q}\right) F\left(\omega_{q}\right)\right\|}\right)$.

Corollary 1 is the vector representation of the above equation obtained by stacking $r_{(i)}^{(l)}\left(\omega_{q}\right)$ and $H_{t_{i}}\left(\omega_{q}\right)$ in order of discretized frequencies $\omega_{q},(0 \leq q \leq(Q-1))$ in $\mathbf{r}_{(i)}^{(l)}$ and $\mathbf{h}_{t_{i}}$, respectively. Matrix $\mathbf{F}$ is diagonal and is of order $Q$ with $F\left(\omega_{q}\right)$ arranged along its diagonal.

Corollary 2. The linear test statistics $\ell_{i}\left(\mathbf{p}_{i}\right)$ of the $M$-ary TR classifier based on observation vector $\mathbf{p}_{i}$ for a single transmitter-receiver pair is given by

$$
\ell_{i}\left(\mathbf{p}_{i}\right)=\Re\left(\frac{\left(\left(\mathbf{F h}_{t_{i}}\right)^{*} \odot \mathbf{h}_{t_{i}}\right)^{H} \sum_{l=1}^{L} \mathbf{p}_{i}^{(l)}}{\sigma_{w}\left\|\left(\mathbf{F} \mathbf{h}_{t_{i}}\right)^{*} \odot \mathbf{h}_{t_{i}}\right\|}\right)
$$

with conditional pdf's

$$
\begin{aligned}
\ell_{i}\left(\mathbf{p}_{i}\right) \mid \mathbb{H}_{0} & \sim \mathcal{C N}(0, L / 2) \\
\ell_{i}\left(\mathbf{p}_{i}\right) \mid \mathbb{H}_{i} & \sim \mathcal{C N}\left(\nu_{i}, L / 2\right)
\end{aligned}
$$

where $\nu_{i}$ equals $L c\left\|\left(\mathbf{F h}_{t_{i}}\right)^{*} \odot \mathbf{h}_{t_{i}}\right\| / \sigma_{w}$.

Proof. Since $H_{t_{i}}$ is scalar and assuming that $\sigma_{v}=0$, Eq. (6) is given by

$$
p_{(i)}^{(l)}\left(\omega_{q}\right)=c_{i}^{(l)}\left(H_{t_{i}}\left(\omega_{q}\right) F\left(\omega_{q}\right)\right)^{*} H_{t_{i}}\left(\omega_{q}\right)+w_{(i)}^{(l)}\left(\omega_{q}\right) .
$$

Therefore, the expression of the log likelihood ratio given in Theorem 1, simplifies to

$$
\begin{aligned}
& \ell_{i}\left(p_{(i)}\right)= \\
& \sum_{q=0}^{Q-1} \Re\left(\frac{\left.\left(H_{t_{i}}\left(\omega_{q}\right) F\left(\omega_{q}\right)\right)^{*} H_{t_{i}}\left(\omega_{q}\right)\right)^{H} \sum_{l=1}^{L} p_{(i)}^{(l)}\left(\omega_{q}\right)}{\sigma_{w}\left\|\left(H_{t_{i}}\left(\omega_{q}\right) F\left(\omega_{q}\right)\right)^{*} H_{t_{i}}\left(\omega_{q}\right)\right\|}\right) .
\end{aligned}
$$

Theorem 2 is the vector representation of the above equation. Both $H_{t_{i}}\left(\omega_{q}\right)$ and $F\left(\omega_{q}\right)$ are stacked with respect to the discretized frequency $\omega_{q}$ in $\mathbf{h}_{t_{i}}$ and $\mathbf{F}$ as explained in the proof of Corollary 1. In addition, $p_{(i)}^{(l)}\left(\omega_{q}\right)$ is stacked in $\mathbf{p}_{(i)}^{(l)}$, for all $\omega_{q},(0 \leq q \leq(Q-1))$. Symbol $\odot$ denotes the Hadamard product.
In both the conventional and TR classifiers, we use the Neyman Pearson test to compute the thresholds $\eta_{i}$ associated with hypothesis $\mathbb{H}_{i}$, for $(1 \leq i \leq(M-1))$, by fixing the probability of false alarm

$$
P_{F}=\int \ldots \int_{\mathbf{z}} \operatorname{Pr}\left(\ell_{1}, \ldots, \ell_{M-1} \mid \mathbb{H}_{0}\right) d \ell_{1} \ldots d \ell_{M-1}
$$

where $\mathbf{Z}=\mathbf{Z}_{1} \cup \ldots \cup \mathbf{Z}_{M-1}$ with $\mathbf{Z}_{i}$ being the observation space associated with hypothesis $\mathbb{H}_{i}$ corresponding to the presence of target $i$.

\section{SIMULATION RESULTS}

In our simulations, the EM channel is probed by

$$
f(t)=\sin \left(2 \pi v\left(t-t_{d}\right)\right) \exp \left(\frac{-\left(t-t_{d}\right)^{2}}{\tau^{2}}\right),
$$

emitted sequentially by individual transducer elements of antenna array $A$. The duration of the signal is constrained to $(0 \leq t \leq 2.5 \mu \mathrm{s})$ and the carrier frequency $v$ is $5 \mathrm{GHz}$. The probing pulse is transmitted into the medium according to the FDTD method [9] obtained by discretization of a simplified version

$$
\begin{aligned}
& \frac{\partial E_{x}}{\partial t}=(1 / \epsilon)\left[\frac{\partial H_{z}}{\partial y}-\left(J_{\text {source }_{x}}+\sigma E_{x}\right)\right] \\
& \frac{\partial E_{y}}{\partial t}=(1 / \epsilon)\left[-\frac{\partial H_{z}}{\partial x}-\left(J_{\text {source }_{y}}+\sigma E_{y}\right)\right] \\
& \frac{\partial H_{z}}{\partial t}=(1 / \mu)\left[\frac{\partial E_{x}}{\partial y}-\frac{\partial E_{y}}{\partial x}-\left(M_{\text {source }_{z}}+\sigma^{*} H_{z}\right)\right]
\end{aligned}
$$

of the Maxwell's equations defined over a 2D Cartesian lattice staggered in time and space. In the above FDTD model, we use the transverse electric (TE) mode with respect to the $z$ axis such that $E_{z}=H_{x}=H_{y}=0$. The nonzero components are $E=\left[\begin{array}{ll}E_{x} & E_{y}\end{array}\right]$ for the electric field and $H_{z}$ for the magnetic field. Notation

$$
J=\left[J_{\text {Source }_{x}}+\sigma E_{x} \quad J_{\text {source }_{y}}+\sigma E_{y}\right]
$$

denotes the electric current density and $M=\left[M_{\text {Source }_{z}}+\right.$ $\left.\sigma^{*} H_{z}\right]$ is the equivalent magnetic current density. Symbols $\epsilon$, $\mu, \sigma$, and $\sigma^{*}$ denote, respectively, the electrical permittivity, magnetic permeability, electric conductivity, and equivalent magnetic loss, which can all vary within the spatial grid. The targets and scatterers are modeled as metal cylindrical rods with very high conductivity values. In our FDTD model, the discretization steps are set to $\Delta x=\Delta y=3 \mathrm{~mm}$, to provide 20 samples per wavelength of the probing pulse. Arrays $A$ and $B$ are considered as linear arrays located parallel to $y$ 
Backscatter of the Probing Signal at Array B

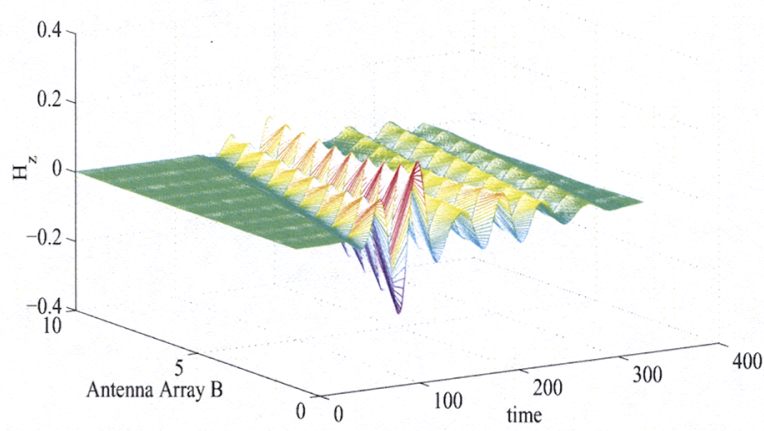

Backscatter of Time-Reversed Field Observed at Array A

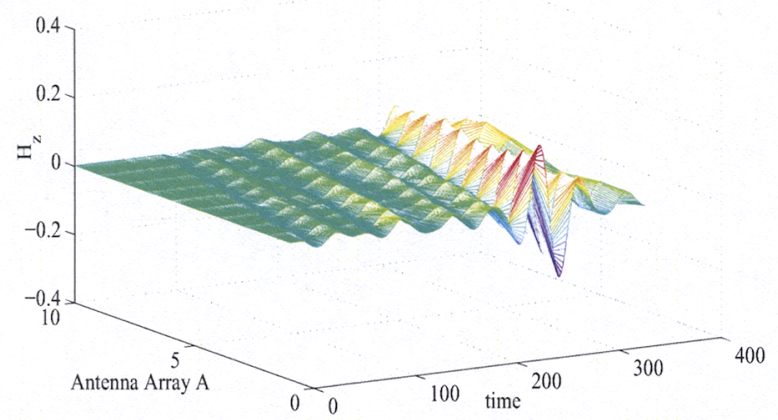

Fig. 2. (Top) Backscattered magnetic fields $H_{z}$ observed at Array $B$, when the first transducer element of Array $A$ probes the channel. These observations are used by the conventional classifier. (Bottom) Magnetic fields $H_{z}$ observed at Array $A$ when the first transducer element of Array $B$ probes the channel with the time-reversed and energy normalized version of its recording made during the forward probing step. The observations shown in the bottom plot are used by the TR classifier.

axis with equal interelement spacing of $\lambda / 4$. Symbol $\lambda$ is the wavelength of the propagating wave, and equals $6 \mathrm{~cm}$. The simulated environment is a $2 \mathrm{D}$ space with dimensions of $15 \times 30 \mathrm{~cm}$, which results in a $50 \times 100$ discretized grid. The locations of the scatterers are randomized during the simulations when the receiver operating characteristics are derived. The computational domain is truncated using the perfectly matched layer (PML) absorbing boundary conditions [10].

In our experiments, we consider the 3 -ary classification problem $M=3$. We use the Neyman Pearson test with the likelihood functions specified in Theorem 1 for the conventional classifier and Theorem 2 for the TR classifier. Thresholds $\eta_{1}$ and $\eta_{2}$ in the Neyman Pearson test are computed by setting the probability of false alarm to 0.01 . The variance of the noise is changed to account for different SNR's. For illustration, Fig. 2 (top) plots the backscattered observations at the transducer elements $(1 \leq p \leq P)$ in antenna array $B$ when the first element $(n=1)$ of Array $A$ probes the EM
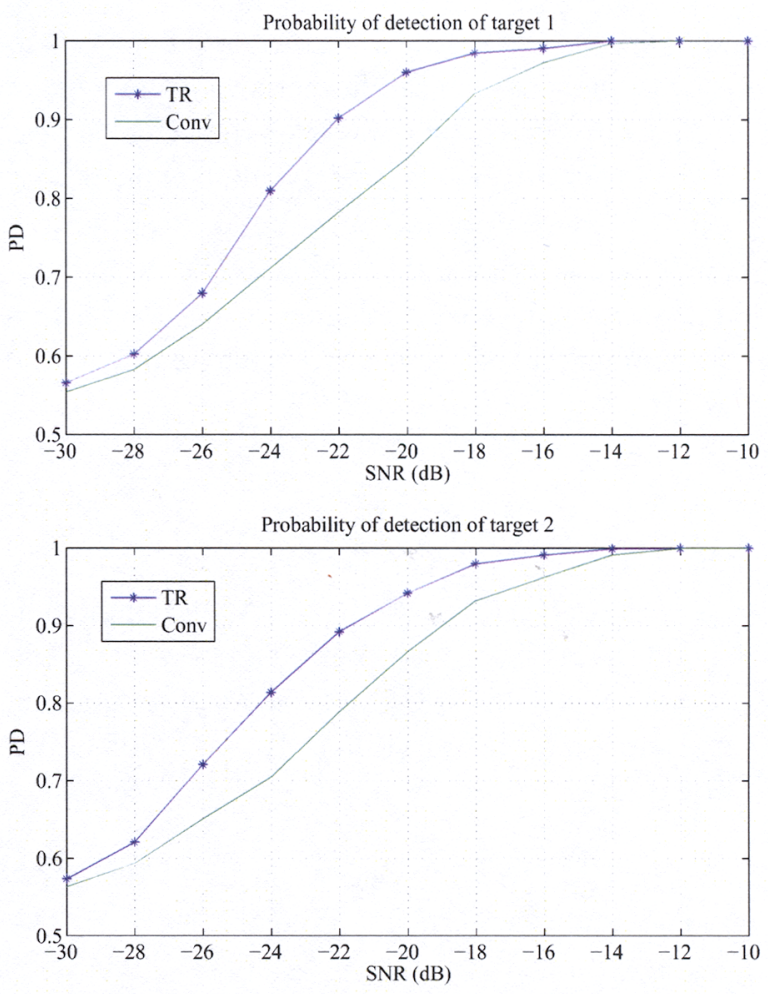

Fig. 3. Comparison of the detection probabilities as a function of signal to noise ratio between the conventional and TR classifiers for targets 1 (top) and 2 (bottom).

channel in the presence of the scatterers and a single target. Note that the observation of all 10 transducer elements in Array $A$ are plotted with the $y$-axis representing the transducer elements in Array $B$. These observations are used by the conventional classifier. Similarly, the bottom plot in Fig. 2 shows the observations at Array $A$ when the fields recorded at the first transducer element in Array $B$ during the forward probing step is energy normalized, time-reversed, and retransmitted back into the medium. Again, recordings of all 10 transducer elements are included in bottom plot of Fig. 2 .

Fig. 3 illustrates the results of the Monte Carlo simulations plotting the receiver operating characteristics (ROC) for the first target (top) and second target (bottom) as functions of the probabilities of detection $P_{D}$ versus the SNR's. For both targets, the TR classifier outperforms the conventional classifier at low SNR's ranging from -14 to $-30 \mathrm{~dB}$. At $P_{D}=0.9$, for example, the TR classifier provides a gain of over $3 \mathrm{~dB}$ with respect to the conventional classifier.

\section{SUMMARY}

The paper derives the $M$-ary TR and conventional ML target classifiers for communications in the EM domain. We used multielement transmitting and receiving antenna arrays 
to derive the ML expressions for the classifiers to detect the presence of the target and to further specify to which of the $M-1$ known target signatures does the detected target belong. The FDTD method is used to simulate the propagation of the EM wave. Our results illustrate the superiority of the $M$-ary TR classification algorithms over conventional classifiers that use only the backscatter of the forward probing signal. In our simulations, we obtain a gain of over $3 \mathrm{~dB}$ for TR classifiers.

\section{REFERENCES}

[1] F. Foroozan and A. Asif, " $N$-Ary Maximum Likelihood Target Detection With Time Reversal," in Proceedings of the IEEE International Symposium on Information Theory (ISIT), Toronto, July 2008, pp. 1873-1877.

[2] M. Fink, "Time Reversal Acoustics," Physics Today, vol. 39, no. 5, 1997, pp. 555-566.

[3] A. J. Devaney, "Time Reversal Imaging of Obscured Targets from Multistatic Data," IEEE Trans. Antennas and Propagation, vol. 53, 2005, pp. 1600-1610.

[4] H. C. Song, W. A. Kuperman, W. S. Hodgkiss, T. Akal, and C. Ferla, "Iterative Time Reversal in the Ocean Reversal," J. Acoustic Society of America, vol. 105, no. 6, 1999, pp. 3176-3184

[5] H. L. Van Trees, Detection, Estimation, and Modulation Theory, vol. I: Detection, Estimation, and Linear Modulation Theory. John Wiley and Sons, 1968.

[6] J. M. F. Moura and Y. Jin, "Detection by time reversal: single antenna,"IEEE Trans. on Signal Processing, vol. 55, 2007, pp. 187-201.

[7] K. S. Yee, "Numerical solution of initial boundary value problems involving Maxwell's equations in isotropic media," IEEE Trans. Antenna Propagation, vol. AP-14, 1996, pp. 302-307.

[8] Mathias Fink, "Time reversal of Ultrasonic Fields Part I: Basic Principles," IEEE Transactions on Ultrasonics, Ferroelectrics, and Frequency control, vol. 39, no. 5, pp. 555-566, 1992.

[9] A. Taflove and S.C Haness, "Computational electrodynamics — the finite-difference time-domain method", second edition, Artech House, Boston, 2000.
[10] G. Mur, "Absorbing boundary conditions for the finitedifference approximation of the time-domain electromagnetic field equations," IEEE Transaction articles on Electromagnetic Compaction 23 , pp. 377-382, 1981. 